\title{
La evaluación del desempeño docente desde la perspectiva de profesores de educación rural
}

\section{Sara del Rosario Castillo-Miranda}

Universidad de Jaén, España srcmooo1@red.ujaen.es

Carolina Hidalgo-Standen

Universidad de La Frontera, Chile carolina.hidalgo@ufrontera.cl

\section{Guillermo Williamson Castro}

orcid.org/0ooo-0001-5313-342X Universidad de La Frontera, Chile guillermo.williamson@ufrontera.cl

\section{Resumen}

Este artículo analiza críticamente las implicaciones de los procesos de evaluación del desempeño docente, desde la perspectiva de los profesores de escuelas rurales multigrado. Utilizando un diseño cualitativo, este estudio recoge la opinión de 56 docentes que trabajan en escuelas rurales de ocho regiones de Chile. Como técnica de recolección de datos se utilizó una entrevista semiestructurada que exploró la opinión docente sobre los instrumentos de evaluación contenidos en el Sistema Nacional de Evaluación del Desempeño Docente ("Docentemas"). Los resultados muestran que a pesar de la aceptación del proceso de evaluación por parte de los profesores, éstos cuestionan la pertinencia metodológica de los instrumentos por considerarlos descontextualizados a la realidad educativa de las escuelas rurales multigrado del país. En conclusión, los procesos nacionales de evaluación docente vigentes, no son adecuados a las escuelas multigrado rurales, ni son bien valorados por los docentes; es necesario contar con procesos apropiados a esta realidad escolar y sus educadores. Por otra parte, los sistemas educacionales y sus diversas expresiones hacia las escuelas deben tener la flexibilidad necesaria para adaptarse a realidades muy diversas que las constituyen.

\section{Palabras clave}

Evaluación de profesores; educación rural, evaluación de la educación enseñanza multigrado Chile (Fuente: Tesauro de la Unesco). 


\title{
Evaluation of Teaching Performance from the Standpoint of Rural School Teachers
}

\begin{abstract}
This study offers a critical analysis of the implications of teacher performance evaluation processes, from the standpoint of multi-grade rural school teachers. Using a qualitative design, opinions were gathered from 56 teachers working in rural schools in eight regions of Chile. As a data collection technique, a semi-structured interview was used to explore what teachers think about the evaluation instruments contained in the National System for the Teaching Performance Evaluation ("Docentemas"). Although teachers accept the evaluation process, the results show they question the methodological relevance of the instruments being used because it is felt they are out of sync with the reality of education at multi-grade rural schools in Chile. In conclusion, the teacher evaluation processes currently being applied nationwide are not suitable for multi-grade rural schools, nor are they well regarded by teachers. Therefore, it is necessary to have processes that are appropriate for this school environment and for those who teach there. On the other hand, educational systems and their diverse expressions in schools must be flexible enough to adapt to the extremely diverse realities that constitute them.
\end{abstract}

\section{Keywords}

Teacher evaluation; rural education; evaluating multi-grade teaching in Chile (Source: Unesco Thesaurus). 


\section{A avaliação do desempenho docente sob a perspectiva de professores de educação rural}

\section{Resumo}

Este artigo analisa criticamente as consequências dos processos de avaliação do desempenho docente, sob a perspectiva dos professores de escolas rurais multiníveis. Utilizando um desenho qualitativo, este estudo coleta opinião de 56 docentes que trabalham em escolas rurais de oito regiões do Chile. Como técnica de coleta de dados, utilizou-se uma entrevista semiestruturada, que explorou a opinião docente sobre os instrumentos de avaliação contidos no Sistema Nacional de Avaliação do Desempenho Docente ("Docentemas"). Os resultados mostram que, embora haja a aceitação do processo da avaliação por parte dos professores, estes questionam a pertinência metodológica dos instrumentos por considerá-los descontextualizados à realidade educativa das escolas rurais multiniveis do país. Em conclusão, os processos nacionais de avaliação docente vigentes não são adequados às escolas rurais multiniveis nem são bem avaliados pelos docentes; é necessário contar com processos apropriados a essa realidade escolar e seus educadores. Por outro lado, os sistemas educacionais e suas diversas expressões quanto às escolas devem ter flexibilidade necessária para adaptar-se a realidades muito diversas que as constituem.

\section{Palavras-chave}

Avaliação da educação; avaliação de professores; Chile; educação rural; ensino multinivel (Fonte: Tesauro da Unesco). 
Chile, desde el año 2014, está desarrollando una Reforma Educacional que considera como uno de sus ejes principales una nueva condición de desarrollo profesional docente a través de lo que se denomina la Nueva Carrera Docente', que supone, entre otros elementos, la revisión de las actuales políticas y procesos de evaluación docente. El Sistema de Evaluación del Desempeño Profesional Docente (EDD) ha sido una de las políticas consideradas centrales en los esfuerzos del Ministerio de Educación para el mejoramiento de la calidad de la educación pública (en Chile, se entiende como red municipal). Sin embargo, la organización gremial docente (Colegio de Profesores de Chile A.G.) se ha opuesto, más que a un proceso de evaluación en sí, al modo, carácter, procesos y procedimientos a través de las cuales se ha desarrollado.

Este artículo presenta parte de los resultados de una investigación doctoral ${ }^{2}$ sobre la percepción de los profesores rurales acerca de la implementación de la EED en escuelas rurales y su desempeño profesional docente. Se muestra que la percepción de los profesores rurales sobre la EED difiere de la percepción de los profesores que se desempeñan en sectores urbanos de nuestro país, en tanto las condiciones físicas, geográficas, culturales y sociales para el desarrollo de la labor docente son diferentes. A través de nuestro trabajo, se indagó por la opinión de 56 profesores que se desempeñan en la educación rural chilena, todos miembros de la Agrupación Nacional de Profesores Rurales ${ }^{3}$ con desempeño en aula multigrado.

1 Ley 20.903. Crea el Sistema de Desarrollo Profesional Docente y modifica otras normas: http://www.ucentral. cl/prontus ucentral2012/site/artic/20160506/asocfile/20160506165206/ppt_sdpd.pdf

2 Tesis doctoral en desarrollo de la autora, en la Universidad de Jaén, Programa de Doctorado en Innovación Didáctica y Formación del Profesorado. La investigación ha focalizado su trabajo en prácticas de enseñanza de profesorado rural chileno en aula multigrado.

3 Con más de 25 años, reúne a un colectivo de profesores de todo el país, con representación en la Mesa Técnica Minis terial para la Educación Rural, compuesta asimismo por el Ministerio de Educación, la Red de Universidades para la Educación Rural.
Adicionalmente, se revisan los modelos de evaluación docente actualmente en uso; se presenta y discute el Sistema de Evaluación del Desempeño Profesional docente, basándose en la Plataforma Docentemas (http://www.docentemas.cl/index.php); se examina la información recabada de los cuestionarios administrados, y finalmente, se analizan las implicaciones de la implementación de este sistema de evaluación en el contexto de la educación rural en Chile.

\section{Debate actual sobre la evaluación docente}

Actualmente existen varias definiciones de la evaluación del desempeño docente (EDD), algunas de las cuales se discuten en este texto. En su mayoría, enfatizan el rol de la evaluación en el proceso de mejoramiento del desarrollo profesional docente, así como también en el control de los resultados obtenidos por estos. De acuerdo con la orEALC/UNESCO, el objetivo de la evaluación del profesorado activo "es mejorar su desempeño, mantenerle motivado o reconocer social y económicamente su trabajo, aunque también considera la evaluación para la selección de puestos directivos. De esta forma, aunque su carácter primordial es formativo, también incorpora elementos de evaluación sumativa" (2007, p. 87).

Si bien en muchos países existe una evaluación de carácter externo al profesorado, entre ellos Chile, otros no lo tienen integrado a sus procedimientos de mejoramiento educacional y considera más bien modalidades de autoevaluación, incluso con carácter informal, como la mayoría de los países de Europa occidental, algunos de Europa del este y algunos latinoamericanos, como Brasil, Ecuador, Nicaragua, Panamá o Paraguay (oreAlc/UnESCO, 2007). La conclusión de un estudio en 50 países de América y Europa es que "no está generalizada la evaluación del desempeño docente de carácter externo" (p. 91), lo que muestra que dicha temática, discutida por el profesorado rural, no es de total consenso y ni siquiera es parte de las plataformas de acción evaluativa como medio para el mejoramiento de la educación, en una amplia variedad y cantidad de países 
ISSN 0123-1294 | e-ISSN 2027-5358 | Educ.Educ. Vol. 20. No. 3 | Septiembre-diciembre de 2017 | pp. 364-381.

Universidad de La Sabana | Facultad de Educación

de ambos continentes. En consecuencia, es posible entonces realizar cambios a partir de las realidades nacionales, ya que la EDD no constituye un parámetro probado universalmente para mejorar el desempeño profesional docente ni, por tanto, la educación.

Se reconoce que el contexto afecta la evaluación del desempeño docente y que, si se reúnen características adversas para el desempeño de su trabajo, ese contexto es calificado por el Estado como de "desempeño difícil"4. Al respecto, Manzi, González y Sub señalan:

La mayor parte del desempeño de los docentes es atribuible a características individuales o de la escuela. Sin embargo, un porcentaje no irrelevante (12\%) del desempeño docente puede ser atribuido a aspectos de la comuna. Este resultado apoya el argumento de que es razonable considerar los aspectos del contexto al momento de analizar los resultados de los profesores en la Evaluación Docente. (2011, p. 188)

Si nos detenemos a revisar ese hallazgo y lo llevamos a la realidad del aula rural, podemos inferir que esta premisa refleja una doble condición adversa: una está en el desempeño, dificultado y reconocido por las condiciones de contexto; la otra viene de las condiciones de trabajo dentro del aula rural, donde existe la realidad de la clase multigrado, en la que se reúnen, como sabemos, más de dos cursos simultáneamente.

La clase es impartida a alumnado que pertenece a diferentes niveles educativos por docente, quien debe potenciar el desarrollo a través del aprendizaje de manera simultánea a cada estudiante en sala. La pedagogía multigrado -como tal y pese a sus complejidades- expresa no una modalidad precaria de

4 Los establecimientos educacionales son calificados de desempeño en condiciones difíciles según su ubicación geográfica, marginalidad urbana, extrema pobreza y dificultades de acceso. Los criterios que se utilizan son los de aislamiento geográfico y ruralidad efectiva, condición del tipo de población atendida y especial menoscabo. Ley 19.070, artículos 118 y 119, DFL No. 1 (Ed)/96 (Estatuto Docente). enseñanza, sino una posibilidad metodológica y conceptual ventajosa para la organización del currículum, la pedagogía, la didáctica y la gestión que se lleva a cabo en escuelas de muchos países, urbanas y rurales, con buenos resultados y aceptación (Williamson, 2010). Pero, y en primer lugar, es necesario considerar el impacto que la propia aplicación de los procesos e instrumentos de evaluación genera en el profesorado, independientemente de sus resultados.

Se ha revisado la validez y confiabilidad de los instrumentos utilizados para la EDD y es reconocida la calidad según el peso cuantitativo de sus resultados (Manzi et al., 2011, p. 61). En cambio los efectos en la plantilla docente evaluada no deben remitirse solo a hallazgos contrastados con los estándares, los cuales se organizan en puntajes asociados a niveles de desempeño. Existe una carga emocional extra para el profesorado que debe someterse a esta evaluación, ya que sus resultados no solo incluyen la retroalimentación para la mejora -que no suele ser el caso, además-, sino que puede tener efectos punitivos ${ }^{5}$, al lograrse el estándar mínimo de aceptación. Sumado a ello, se incorpora el juicio social -incluso de sus pares-a que es sometido el profesorado. Todo esto incidirá de manera significativa en la predisposición que se tenga al trabajo evaluativo.

La calidad de una situación de evaluación se verá alterada en sus resultados, si el evaluado trabaja bajo presión. El entorno que rodea a la situación de evaluación también ejercerá un rol fundamental a la hora de someterse a la EDD, de modo que-como plantea Bonvecchio (2006)- el clima que se genera

5 Artículo 1b: "Los profesionales de la educación que resulten evaluados con desempeño insatisfactorio, deberán someterse a nueva evaluación al año siguiente conforme a los planes de superación profesional que determine el reglamento. Si en la segunda evaluación el resultado es nuevamente insatisfactorio, el docente dejará la responsabilidad de curso para trabajar durante el año en su plan de superación profesional, debiendo el empleador asumir el gasto que representa el reemplazo del docente en el aula. Dicho docente será sometido al año siguiente a una tercera evaluación. Si el desempeño en un nivel insatisfactorio se mantuviera en la tercera evaluación anual consecutiva, el profesional de la educación dejará de pertenecer a la dotación docente". 
antes y durante su aplicación, en términos de serenidad, confianza, buena disposición, o sus contrarios, afectará considerablemente a los profesores y profesoras sometidos a evaluación. Esta se ve como una condición externa al instrumento aplicado, que será un factor que altere la confiabilidad del instrumento, ya que, para que sea confiable, debe presentar consistencia en la construcción del instrumento y en su administración.

El profesorado tiene diferentes estilos de enseñar y es portador de saberes propios y creencias relacionadas con la construcción de la identidad docente, sin dejar de mencionar la importancia de su formación inicial. En ese contexto, se considera que los instrumentos que componen la EDD no son pertinentes para la recogida de información fidedigna del desempeño docente en el aula rural.

La Organización para la Cooperación y el Desarrollo Económicos (OECD), por su parte, dice promover políticas y programas asociados a diversas modalidades de evaluación de desempeño docente, asociado a un mejoramiento de su quehacer profesional, como condición de desarrollo y mejoramiento continuos de la educación. Organizaciones como esta parten de que el profesorado cumple un rol esencial en mejorar el aprendizaje del alumnado y que el efecto de una buena docencia es acumulable en la vida de quien estudia (Sanders y Horn, 1998; Sanders y Rivers, 1996). Por esta razón las políticas educativas han enfatizado en el desarrollo de programas que fortalezcan la calidad docente y mejoren su efectividad, lo que se refleja en el incremento de sistemas de evaluación de la docencia. De hecho, de acuerdo con la OECD, actualmente la gran mayoría de países que conforman esta organización cuenta con leyes para regular los procesos de evaluación de su profesorado (Murillo, González y Rizo, 2006; OECD, 2013a).

Dos propósitos son reconocidos como los más importantes en esta evaluación (OECD, 2013b; Peterson, 2000; Stronge y Tucker, 2003). El propósito formativo ofrece al docente la oportunidad para aprender durante el curso de la evaluación sobre las fortalezas y debilidades de su práctica. El foco de la evaluación es que el profesorado reflexione y aprenda sobre su práctica pedagógica y de esta manera mejore su quehacer pedagógico, en beneficio de potenciar el aprendizaje del alumnado. Por otra parte, el propósito de control (accountability) está centrado en "hacer a los profesores responsables" por los resultados de aprendizaje del estudiantado. Esta evaluación sumativa se asocia a consecuencias en el desarrollo profesional docente. Por ejemplo, si el profesorado tiene un buen desempeño, recibirá incentivos por esta dimensión, como aumento en su remuneración, bonos o avances en su carrera profesional. Sin embargo, si los resultados de la evaluación son negativos, puede perder beneficios económicos o incluso ser marginado de la institución educativa (OECD, 2013, p. 16). Además, algunos sistemas de evaluación reportan objetivos mixtos de formación y control (Stronge y Tucker, 2003). Para la OECD (2009; 2013), la combinación de estos propósitos crea dificultades y pone en riesgo el buen desarrollo del proceso evaluativo. Por ejemplo, la reflexión y aprendizaje del profesorado se ven amenazados cuando los resultados de la evaluación pueden conllevarles consecuencias negativas.

En relación con los modelos de evaluación docente, tres son descritos como los más utilizados: Modelo de Certificación, Modelo de Evaluación de Desempeño, Modelo de Valor Agregado. Cada uno tiene diferentes concepciones de lo que significa calidad docente y propone diferentes métodos para medirla (Goe, Bell y Little, 2008; Goe, 2007). El Modelo de Certificación parte de la base de que existen ciertas características docentes, personales y profesionales, que favorecen el logro de aprendizaje de los estudiantes. Algunas de estas características son: 1) antecedentes académicos del profesorado, por ejemplo puntajes en pruebas estandarizadas, pruebas de selección universitaria, pruebas de conocimiento pedagógico y disciplinar posgraduación, 
entre otros; 2) antecedentes de desarrollo profesional, por ejemplo asistencia a diplomados, cursos, ayudantías, y 3), experiencia profesional. Esta forma de evaluación docente es usada principalmente para facilitar la toma de decisiones para contratación, asignación de incentivos y otras decisiones de la carrera profesional (Goe et al., 2008).

El Modelo de Evaluación de Desempeño sugiere que la calidad del profesorado está determinada por las acciones que utilizan fuera y dentro de la sala de clases para mejorar el aprendizaje del alumnado. Este modelo se basa en la selección de criterios de calidad docente que se evalúan en función de determinados estándares. Estos criterios deben ser claramente definidos, consensuados y comunicados entre profesorado, estudiantes con liderazgo escolar y comunidad en general. El claustro es medido y evaluado en el grado con el cual cumplen con cada uno de los criterios definidos en función de determinados estándares. Una de las principales ventajas de este sistema es que entrega información directa al profesorado y, además, que el uso de estándares unitarios favorece cierta comparación y, por tanto, apunta a la generación de sistemas de promoción e incentivo (Darling-Hammond, 2013; Howard y Gullickson, 2010; Coggshall y Basset, 2008).

Finalmente, el Modelo de Valor Agregado está basado en la idea de que la calidad docente está determinada por el nivel de productividad (efectividad) docente en el incremento del aprendizaje del estudiantado. Este incremento se determina a través del análisis estadístico del resultado que se obtiene en pruebas estandarizadas. Este modelo requiere para su implementación la generación de gran cantidad de datos relacionados con el desarrollo de cada estudiante en un determinado periodo de tiempo y el desarrollo de modelos estadísticos complejos que permitan el procesamiento y análisis de estos datos (Campbell, Kyriakides, Muijs y Robinson, 2003; Goe, 2007; Lewis y Young, 2013; Goldhaber, 2008). Estos dos últimos requisitos hacen que este modelo aún no esté ampliamente difundido.
En América Latina, y particularmente en Chile, el modelo de evaluación docente está vigente desde el año 2003 y se hace obligatorio el año 2005. Este sistema de evaluación se ha ido perfeccionando a través del tiempo. Nace de una negociación tripartita entre Colegio de Profesores, el Ministerio de Educación ${ }^{6}$ y la Asociación Chilena de Municipalidades7. Este método de recolección de información del trabajo docente es el instrumento actual por el cual son medidos en su desarrollo profesional quienes trabajan en colegios y liceos municipales de todo Chile (Ávalos, 2004; Bonifaz, 2011; Taut, Santelices, Araya y Manzi, 2010).

La Ley 19.961 sobre evaluación docente la define como un sistema de evaluación de profesionales de la educación que se desempeñen en funciones de docencia de aula, de carácter formativo, para mejorar su labor pedagógica y a promover su desarrollo profesional continuo, según lo establecido en el artículo 70 del Decreto con Fuerza de Ley No. 1 de 1996 del Ministerio de Educación, con niveles de desempeño que corresponden a destacado, competente, básico o insatisfactorio ${ }^{8}$.

6 El Centro de Perfeccionamiento, Experimentación e Innovación Pedagógica (CPEIP) se encarga de su elaboración, aplicación y seguimiento. Como organismo perteneciente al Ministerio de Educación, se encarga desde el año 2003 del diseño, administración e implementación de la EDD.

7 En Chile se considera -en general, aunque está en discusión-el sistema municipal de educación como el heredero de la tradicional escuela estatal y pública republicana, que duró hasta el año 1980.

8 Portal Docentemás (http://www.docentemas.cl/dmo1 marcolegal.php). Los niveles de desempeño estipulados en el Reglamento sobre Evaluación Docente son los siguientes: Destacado: indica un desempeño profesional que clara y consistentemente sobresale con respecto a lo que se espera en el indicador evaluado. Suele manifestarse por un amplio repertorio de conductas respecto a lo que se está evaluando, o bien, por la riqueza pedagógica que se agrega al cumplimiento del indicador. Competente: indica un desempeño profesional adecuado. Cumple con lo requerido para ejercer profesionalmente el rol docente. Aun cuando no es excepcional, se trata de un buen desempeño. Básico: indica un desempeño profesional que cumple con lo esperado en el indicador evaluado, pero con cierta irregularidad (ocasionalmente). Insatisfactorio: indica un desempeño que presenta claras debilidades en el indicador evaluado y estas afectan significativamente el quehacer docente. 
Quien es evaluado queda sometido a los efectos de cuestionamientos tanto sociales como personales, lo que impacta de manera significativa en sus futuras prácticas de aula. Álvarez (2000) plantea que entre el profesorado este es un aspecto importante a enfrentar cuando se busca mejorar la calidad educativa.

\section{Surge el problema}

En este contexto de la implementación de la evaluación docente en Chile, los profesores y profesoras de escuelas rurales multigrado enfrentan los procesos de EDD en una particular posición territorial, pedagógica y profesional, en zonas de alta vulnerabilidad y relativo o difícil acceso. Como lo plantean Porlán y Rivero (2000), el conocimiento docente conlleva los saberes académicos, los saberes basados en la experiencia, las rutinas y guiones de acción y las teorías implícitas. Todo ello da cuenta de la singular práctica en un aula multigrado, la que no es validada por el Estado regularizador, que interpone la necesidad del trabajo estandarizado, lo cual visibiliza las necesidades del estudiantado rural y las que vienen de las peculiaridades del contexto sociocultural de la localidad en la que se encuentra la escuela.

El profesor o profesora rural está en condiciones diferentes de las de sus pares de ciudad, y puesto que las que exige la EDD no están dadas en el aula rural, sea esta multigrado o combinada' ${ }^{9}$, se llega a que esta medición no considera la realidad de este tipo de escuelas. Manzi et al. (2011), recabando investigaciones anteriores -y citando a Eisenberg (2008)-, enfatizan en la importancia de considerar el contexto escolar a la hora de emitir juicios sobre las evidencias recogidas para la evaluación (EDD). El profesorado rural en Chile, históricamente, se han constituido como un colectivo con identidad propia, expresada en organizaciones que han podido

9 Se entiende como aula multigrado o combinada la que re úne físicamente a la totalidad de los niños y niñas de la escuela, sin distinción de grados, en una sala o que a lo menos fusiona dos más grados. articular la defensa y conquista gremial con la propuesta técnica (Williamson, 1999). Sin embargo, la producción de conocimiento en Chile acerca de los efectos o implementación de este sistema de evaluación en sectores rurales es casi inexistente. Del mismo modo, los instrumentos de evaluación tradicionales, como el Marco para la Buena Enseñanza, no establecen ningún indicador que permita identificar diferencias e individualizar las características del aula multigrado y las particularidades que adquiere la enseñanza.

El profesorado que se desempeña en aula multigrado constituye la principal preocupación de este artículo, ya que una lectura rápida del estado del arte sobre el tema muestra que la presencia y preocupación por este sector específico es precaria. En general, de modo explícito o implícito, hay un sesgo urbano y de escuelas completas en la política educativa y en las declaraciones de propósitos y discursos públicos al respecto de la EDD. Así lo sostenemos, al revisar los instrumentos que son aplicados en esta evaluación.

\section{Metodología de estudio}

Este artículo presenta una reflexión crítica aplicada a la evaluación de desempeño de profesores rurales para generar insumos con que enfrentar los nuevos desafíos para la educación de las poblaciones rurales y la nueva carrera docente que forma parte de la reforma educacional que se impulsa en Chile desde el año 2014. La investigación principal ha recogido información directa de campo, de docentes rurales en aula multigrado o combinada, teniendo como requisito el que hayan sido sometidos a la EDD. Se trata de revisar las opiniones de los profesores y profesoras rurales respecto de este proceso y extraer de ellas algunas consideraciones que puedan contribuir, tanto a comprender los procesos actuales como a generar alertas e ideas para las futuras definiciones referidas a la nueva carrera docente, definida en la reforma educacional. 
La presente investigación es de tipo exploratorio. Se aplicó un cuestionario semiestructurado, que se validó debidamente por expertos (García y Cabero, 2011; Rodríguez, Hoffmann, Mackedanz y Hoffmann, 2011) y que contestaron online 19 profesores(as) de diversas regiones y en forma presencial otros 37 , durante la muestra de Cueca Nacional, realizada en Linares, Región del Maule, en octubre 2014, actividad a la que asisten docentes de todo Chile. Este profesorado no corresponde a una muestra, sino a un grupo seleccionado de acuerdo con la determinación intencionada de la investigadora. Por ello, no se considera ninguna variable de género, edad, origen étnico o lugar de habitación, sino que, en función de los propósitos del estudio, se consideran dos exigencias básicas: 1) haber participado en la Evaluación o estar en ese proceso en el momento de la consulta escrita, en el sistema de EDD, y 2) ser docente rural y trabajar en aula multigrado.

El cuestionario aplicado constó de ítems cerrados en el que debían inclinarse por un nivel de adscripción a lo declarado. Cada sección del cuestionario se relacionó de manera directa con cada instrumento evaluativo de la EDD, a saber: autoevaluación; entrevista con un par evaluador; informe de terceros; y desarrollo del portafolio, que consta de dos partes: una escrita y la otra de clase filmada. Los ítems se completaron con una pregunta abierta, la que permitió al profesorado profundizar en las declaraciones anteriores.

\section{Análisis e interpretación de resultados}

El sistema de evaluación de desempeño docente se organiza a partir de cuatro situaciones de evaluación, las cuales abarcan la planificación, organización, implementación y reflexión sobre la práctica docente. Los instrumentos son cuatro: cuestionario de autoevaluación, entrevista de evaluación par, informe de referencia de terceros y portafolio de desempeño pedagógico, como se indicó. De manera similar, cada uno de ellos da cuenta de la relación con los dominios, criterios y descriptores propuestos en el Marco para la Buena Enseñanza (MBE). A con- tinuación se presentan los principales resultados de este estudio, organizados considerando la opinión del profesorado en relación con los cuatro instrumentos descritos.

\section{Cuestionario de autoevaluación}

Se trata de un instrumento que aborda el desempeño docente desde la perspectiva de quien es evaluado, como puede entenderse. Este instrumento se estructura en una serie de ítems, idénticos para quienes participan, derivados del MBE y que están orientados a que cada docente reflexione acerca de su propia práctica y, a partir de dicha reflexión, evalúe su desempeño (Manzi et al., 2011). El objetivo de este instrumento se cumple y quien responde no suele cuestionar una buena calificación. Pese a ello, la mayoría de participantes del estudio -personalmente o en línea- siente que las preguntas no están relacionadas con la realidad específica de la escuela rural, como veremos. Esto se debe a que los cuestionarios son únicos, por estar elaborados por el sistema central, en el que predomina una visión homogénea nacional, mayoritaria, genérica y urbana. Lo anterior se aprecia en la tabla 1.

Además de emitir un juicio acerca de su propio desempeño y explicar la elección -solo si es destacado el logro máximo del estándar-, cada criterio de este ítem presenta tres indicadores, de los cuales -según arroja su revisión- uno o dos son extrapolables a la clase multigrado y el otro o los otros no se enmarcarían en esta realidad. Respecto a la optimización del tiempo, su adecuación es relativa, ya que el profesorado con grupo multigrado no siempre puede iniciar la clase a la vez en todos los cursos. Como sabemos, se debe trabajar con diversas estrategias, atendiendo a los diferentes grupos dentro de la sala; por tanto, no se evidencia su trabajo con el global de sus estudiantes de forma continua hasta el término de la sesión. Así, se atiende a los grados indistintamente y al curso en el que se evalúa en diferentes momentos. Si se dedicara solo al grupo que está siendo evaluado, debería abandonar los otros cursos que hay en la misma sala de clases. 


\section{Tabla 1. Autoevaluación}

\begin{tabular}{|c|c|}
\hline \multicolumn{2}{|c|}{$\begin{array}{l}\text { Reflexiones acerca del grado en que cada uno de los siguientes indicadores forma parte } \\
\text { de su práctica docente habitual. }\end{array}$} \\
\hline & $\begin{array}{l}\text { Comienzo mis clases puntualmente y trabajo con todos mis estudiantes de forma } \\
\text { continua hasta el término de estas. }\end{array}$ \\
\hline $\begin{array}{l}\text { Criterio C-4: Optimiza el tiempo } \\
\text { disponible para la enseñanza }\end{array}$ & $\begin{array}{l}\text { Aprovecho el tiempo de clase en actividades relevantes para el logro de los objetivos } \\
\text { de aprendizaje. }\end{array}$ \\
\hline & $\begin{array}{l}\text { Adapto los tiempos de las actividades de acuerdo a los avances y dificultades que } \\
\text { observo en mis alumnos. (Gobierno de Chile, s.f., p. 14) }\end{array}$ \\
\hline
\end{tabular}

\section{Entrevista de evaluación par}

Este instrumento permite recoger información sobre el desempeño docente desde la perspectiva de un similar, que desempeña su trabajo en condiciones equiparables a quien se evalúa, y que se denomina "Evaluador par". Para cumplir este rol, el profesorado se postula voluntariamente y participa de un proceso de selección, capacitación y entrenamiento que pretende habilitarlo para aplicar correctamente el instrumento. Los evaluadores pares son docentes del sector municipal con al menos cinco años de experiencia profesional docente, preferentemente trabajan en la misma comuna de quien es evaluado y deben desempeñarse en su mismo nivel, sector y modalidad de enseñanza (Manzi et al., 2011, p. 48).

Cada año, el Centro de Perfeccionamiento, Experimentación e Investigaciones Pedagógicas (CPEIP) selecciona los docentes que capacita el equipo de Docentemás ${ }^{10}$. Todo el profesorado que hemos entrevistado hace referencia a que la realidad de la escuela rural y la sala multigrado o combinada no se considera en las preguntas que se les formulan en este ítem.

En nuestros resultados, se considera unánimemente que el valuador par no conoce la realidad de

10 Equipo Docentemás, por encargo del CPEIP, se constituye en el Centro de Medición de la Universidad Católica de Chile (MIDE-UC) y es responsable de desarrollar las distintas acciones y materiales que requiere el proceso de evaluación. Trata aspectos tanto técnicos como operativos, facultad delegada por el Ministerio de Educación. la sala multigrado. Y es que estos "pares" no son tales, al no haber docentes de modalidad de escuelas rurales multigrado que trabajen niveles y grados en contextos multinivel y multigrado. Los docentes de la muestra manifiestan que muchas veces se actúa con indiferencia al contexto de la escuela, al consultárselos por las características que debe tener quien evalúa, la mayoría apuntó al clima que debe tener la entrevista y a comentarios de la persona que dirige el proceso. Esto se ejemplifica en la opinión expresada en comunicación personal por una profesora de la región de Biobío, respecto de la necesidad de "ser empático para evaluar en un contexto del aula rural, no venir a dar ejemplos de cómo se hacen las cosas sin conocer la realidad" (consulta escrita, octubre 2014). Consideran, por tanto, que las preguntas no han sido pertinentes. No obstante, es necesario indicar que, al revisar la pauta de la entrevista, una parte importante de lo consultado en la misma refiere a contextualización de la enseñanza. El hecho de que las personas evaluadas no estén de acuerdo con este instrumento nos lleva a inferir al menos que el clima aludido no fue el idóneo o el instrumento no fue suficientemente explicado y el profesorado motivado a expresarse en sus propios términos.

\section{Informe "Referencia de terceros: la heteroevaluación"}

Este instrumento recoge la apreciación de la superioridad jerárquica sobre el desempeño profesional docente. Aquí se espera que dirección y jefa- 
ISSN 0123-1294 | e-ISSN 2027-5358 | Educ.Educ. Vol. 20. No. 3 | Septiembre-diciembre de 2017 | pp. 364-381.

tura de la Unidad Técnico Pedagógica (UTP) de los centros escolares, quienes tienen la oportunidad de observar y conocer el trabajo del profesional en la escuela, puedan calificar su desempeño. Como expresan Manzi et al., "el superior jerárquico debe, basándose en su conocimiento del trabajo del profesor, calificar su desempeño en cada uno de los ámbitos que se abordan empleando los cuatro niveles de desempeño del sistema (Destacado, Competente, Básico e Insatisfactorio)" (2011, p. 47). La jerarquía UTP debe reflexionar en el desempeño docente sometido a evaluación e identificar fortalezas y debilidades, para ser indicadas en los cuestionarios a completar. Por ello se considera que, por su ubicación en el organigrama, conoce en forma directa y constante el trabajo educativo y el contexto en que se realiza.

El profesorado consultado, en referencia a este instrumento evaluativo, considera que la selección realizada para determinar a la persona que emitió el juicio sobre su trabajo en sus labores cotidianas no fue adecuada. Como muestra, en muchos casos, quien evaluó o completó el informe nunca había visitado la escuela rural a la que pertenecía el profesorado evaluado. También se manifiesta en reiteradas ocasiones que se completa el informe en la formalidad de un puesto de trabajo, ya que, en la mayoría de los casos, se mencionan las oficinas del Departamento de Administración de Educación Municipal (DAEM), ubicadas a muchos kilómetros de distancia de la realidad educativa que se pide evaluar. Es por ello que este instrumento se considera inapropiado, ya que no se llega a las fuentes para completar los indicadores que considera esta evaluación.

Por todo ello, el profesorado de nuestro estudio recomienda, para acceder a las fuentes de información" ${ }^{11}$ poder emitir juicios sobre el trabajo docente, recopilar en la escuela información de: observación directa de clases, revisión de documentos de trabajo en la escuela e interacción con la comunidad educa-

11 Sugerencias para el informe de terceros, publicadas en el Portal Docentemás 2014-2015. tiva. Ante la pregunta abierta referida a cuál sería el mejor sistema para mejorar la evaluación de terceros, el profesorado consultado responde que los aspectos indicados en el portal de información del CPEIP, pero que no se lleva a cabo realmente. Ante la pregunta sobre los datos entregados en el informe de terceros, un profesor de la región de Coquimbo dice:

Para que sea más válido, se deben considerar varias instancias que dé un informe del trabajo que se realiza en forma directa; eso puede ser encuesta a estudiantes, de apoderados y pares que entreguen apreciaciones del trabajo. Finalmente, poder recibir sugerencias metodológicas para la mejora de la práctica de cada docente evaluado. Acá llegan a hacer preguntas que ni siquiera ellos comprenden para aplicar a la sala multigrado. (Comunicación personal, consulta escrita, octubre 2014)

\section{El portafolio}

Este instrumento se divide en dos productos: 1) la propuesta escrita para desarrollar una unidad didáctica, detalles de su implementación y evaluación, todo ello desarrollado y escrito, siguiendo pautas determinadas por el instrumento; 2) una grabación de una clase previamente planificada de 40 minutos exactos, que es registrada por personal externo, desconocido por quien está en evaluación. El camarógrafo filma y entrega la grabación al docente filmado, en sobre sellado, y luego el profesor entrega todas las evidencias de su proceso evaluativo, también en sobre cerrado, a su administración educacional comunal correspondiente.

CONFECCIÓN DEL PORTAFOLIO: Esta parte de la evaluación -que recaba, recupera y presenta información acerca del trabajo práctico del docente-está a cargo de quien se somete a la misma. Sobre el papel, es un instrumento que recoge evidencias directas de las formas que este tiene de comprender su práctica, de prepararla, aplicarla y, desde ahí, de construir nuevos significados para su mejora. Este instrumento permite recoger muestras directas o evidencias concretas 
del quehacer de cada docente y posibilita que este dé cuenta de su desempeño en las acciones profesionales centrales del trabajo en el aula. Se estructura en dos módulos: uno en que se presenta información referida al diseño, implementación y evaluación de una unidad pedagógica, y otro que consiste en la filmación de una clase. En cada módulo se incluyen determinados productos (Manzi et al., 2011, p. 49).

Al respecto, el profesorado consultado opina que los aspectos considerados en el portafolio, es decir, las áreas que evalúa, tampoco son representativos de la realidad de la escuela rural multigrado o con cursos combinados. En cuanto a la planificación exigida, declaran que se escribe sin dificultades, pues siguen la pauta de lo que se les pide. Pero el periodo a planificar es de 8 horas pedagógicas, sin modificarse atendiendo a la realidad de la sala multigrado (el tiempo real en sala se debe compartir con otros cursos, lo que no se declara), ni tampoco se considera o visibiliza en las tareas solicitadas en la pauta para completar el portafolio. Del mismo modo, se opina que la evaluación de la clase programada se realiza sin dificultad, ya que considera al curso en que se realiza la evaluación docente. No obstante, en el estudio de campo que hemos realizado, las entrevistas en profundidad señalan la evaluación y la planificación como los principales problemas al impartir enseñanza en la clase multigrado' ${ }^{12}$.

En lo referido al trabajo con apoderados, el portafolio incorpora preguntas de desarrollo dirigidas a la comprensión de la tarea de enseñar y mejorar sus prácticas. Un ítem está orientado a la comprensión de la evaluación desarrollada: se pregunta acerca del apoyo de la familia y la retroalimentación del proceso de aprendizaje de la infancia en casa. El profesorado nos menciona que la realidad de la escuela no lo permite, pues muchas familias no entienden la mayor parte de los contenidos. Así, las reuniones de apoderados son para coordinar mucha información

12 Trabajo de campo, Investigación Doctoral de Sara Castillo, localidad de Paredones, Chile. de los diferentes cursos, por lo que no se les puede pedir apoyo del tipo que describe el portafolio como situación ideal. Es lo que muestra el comentario de un profesor de la región de O’Higgins: “En una parte me preguntaron si los papás entendían de la retroalimentación. Qué voy a decir, si apenas me apoyan con las tareas, ellos, no sé si será por la cercanías entre las familias, pero acá mis apoderados fueron mis alumnas. Entonces yo sé lo que les puedo pedir" (comunicación personal consulta escrita, octubre 2014).

La mayor parte del profesorado consultado manifiesta que el trabajo de la evaluación de desempeño docente es un proceso solitario, no tienen posibilidades de realizar preguntas a sus pares más cercanos que ayuden al desarrollo del trabajo. Esto lo ilustran dos profesoras, así:

No es una instancia que permita ser una herramienta adecuada que refleje el nivel real de docentes en el aula, por la forma que se aplica, y los módulos no reflejan el quehacer educativo de un docente en el aula rural; además nosotros no tenemos forma de preguntarle a otro colega que ya se evaluó. A mí me toma bajar a Tenox 25 minutos y para llegar a Lidux casi media hora, ¿a qué hora pido ayuda? (región de Coquimbo, comunicación personal, consulta escrita, octubre 2014).

El trabajo de un docente en el aula rural es diferente, en forma y estructura, al de los docentes urbanos. Somos solitarios. El bagaje cultural del contexto rural influye en la respuesta de la aplicación de los portafolios que corresponde completar (región de Los Lagos, comunicación personal, consulta escrita, octubre 2014).

En síntesis, contamos con numerosas evidencias de que la realidad de la escuela rural no es reflejada en las características que adquiere la declarada en la EDD, especialmente en la aplicación práctica.

Clase fILMADA: Se trata de un acercamiento a la realidad de las clases que se imparten; sin embargo, como su intención es filmar solo lo requerido por 
ISSN 0123-1294 | e-ISSN 2027-5358 | Educ.Educ. Vol. 20. No. 3 | Septiembre-diciembre de 2017 | pp. 364-381.

Universidad de La Sabana | Facultad de Educación

la pauta de elaboración del portafolio, aquí la clase escapa de lo solicitado por esta pauta y se pierde lo singular de una clase multigrado.

La filmación ha demostrado ser uno de los elementos que aporta más información al proceso de evaluación, en la medida que da cuenta de lo que sucede en la sala de clases y en la interacción del profesor con los estudiantes. Desde esta perspectiva, se ha considerado necesario construir una pauta de observación complementaria, que permita indagar en aspectos que están insuficientemente representados en la escala de evaluación original. (Milicic, Rosas, Scharager, García y Godoy, 2008, p. 81)

En la página Docentemas ${ }^{13}$ se publican indicaciones a tomar en cuenta para los días previos y para el día de la grabación. Llama la atención la sugerencia de poner un cartel en la puerta cuando se está filmando y que se debe indicar el alumnado eximido, que no participan de la clase filmada, para evitar que la cámara los registre. Ello, no hay que olvidarlo, en un contexto nacional en Chile de movilizaciones sociales y educacionales contra la discriminación.

En toda la investigación realizada, este ítem aparece como el que presenta los mayores problemas, pues son múltiples las dificultades mencionadas por el profesorado. Todas surgen en el momento en que se realiza la filmación. El curso multigrado no se divide para realizar el registro filmado. El profesorado evaluado tiene que estar con toda la infancia de diferentes cursos y, al realizarse la filmación, debe atender las necesidades de todos sus estudiantes, ya que está impartiendo la enseñanza para todo el grupo. Como sabemos, cuando la infancia se encuentra con situaciones novedosas no escapa a su necesidad de indagar y experimentar con lo nuevo, además de su natural afinidad por el juego. Esto lo ilustra una comunicación personal de una profesora de región

13 Sitio ( $h t t p: / / w w w . d o c e n t e m a s . c l /)$ destinado a mantener toda la información acerca de la evaluación docente, la publicación de la información entregada está a cargo del CPEIP. del Maule: "Parece que lo hacen peor: sabían que me estaban filmando, yo había ensayado con los dos [grupos] que tengo en cuarto, y salieron preguntando todos. Por supuesto, les contestaba, pero me ponía nerviosa. La clase se alteró. Yo creo que [esto] me bajó mi evaluación" (consulta escrita, octubre 2014). Como se ve, la EDD exige planificar la enseñanza y que se la filme partiendo de que se trata de un solo curso, pero en realidad no lo es y son diferentes las tareas que se deben estar supervisando a la vez y en diferentes grados al mismo tiempo.

La pauta de "corrección" de filmaciones indica entre sus ítems principales el verificar la enseñanza para todos y todas, y eso se visualiza observando que todo el grupo trabaje en lo mismo y con los mismos materiales. Esta tarea es muy difícil de conseguir en la enseñanza multigrado, con concepción curricular integradora, porque no se la considera en el proceso al organizarse por asignaturas y un niño de primer grado no trabaja con los mismos materiales y exigencias que uno de cuarto grado. Las pautas de observación de videos están construidas en especial para monitorear las tareas semejantes entre discentes, que deben seguir las indicaciones de la clase, respetando lo presentado en la planificación entregada para la clase filmada. Principalmente se verifica la Dimensión C, del Manual para la Buena Enseñanza (MBE), que define cómo se implementa la enseñanza para todo el alumnado (Gobierno de Chile, s.f., pp. 27-32).

\section{Conclusiones}

El sistema educativo chileno ha instalado sistémicamente formas de mantener una determinada supervisión y control sobre la totalidad del profesorado municipal. Son procedimientos que arrojan cifras que le permiten al Estado monitorear determinado enfoque de calidad docente en ejercicio. Se parte de una concepción definida con un enfoque positivista de estándares, definidos por el Estado con criterios técnicos, expresión de una teoría curricular universalista que desconoce la diversidad en todas 
sus manifestaciones, y a las que no dota de medios y recursos específicos para atender el territorio y el carácter curricular y pedagógico inherente a la escuela multigrado. En consecuencia, las cifras que se entregan como promedios o resultados del proceso no son veraces, ya que solo evidencian lo que se reconoce oficial, hegemónica y universalmente como realidad absoluta de la escuela chilena, que se persigue evidenciar y validar a través de una evaluación encorsetada.

El sistema no admite objeción a este proceso, ya que negarse a participar en él trae consecuencias negativas para la carrera docente de cada persona. Nuestra investigación arrojó, como se ha indicado, que el profesorado rural no considera apropiados los instrumentos que se les aplica en la EDD. A pesar de ello, participan obligados de este sistema de evaluación, que reporta "evidencias" en imposible comparación con estándares nacionales que no establecen diferencias en el ejercicio docente, al considerar formalmente que un curso monogrado es comparable estadística, social y pedagógicamente con otro multigrado.

En opinión de nuestro profesorado, los instrumentos que componen la EDD no son pertinentes ni adecuados a la realidad rural multigrado, pues la evaluación se dirige a un curso y un grupo etario común: las condiciones que exhibe la sala, que reúne a infancia de diferentes cursos, no está consideradas en muchas de las exigencias de desempeño orientadas por los estándares que se utilizan para implementar la EDD.

Para el sistema lo importante es verificar la consecución de estándares de desempeño docente homogéneos y urbanos, no interesándose en conocer las problemáticas surgidas del acto educativo en sus múltiples presentaciones. Así, los profesores y profesoras contribuyen al juego dominante de la estandarización y universalización de la evaluación docente, pero en sus opiniones manifiestan constantes críticas a todo el sistema, en el proceso y en sus instrumentos particulares, que no parecen ser atendidas.

Vista desde los márgenes del sistema educativo -por el carácter minoritario de lo rural-, una de las principales deficiencias del Sistema de Evaluación Docente está en su incapacidad de reconocer que la labor docente es heterogénea y, por ello, que un sistema universal no puede alcanzar la riqueza de la diversidad de prácticas, identidades y realidades de dinámicas curriculares y pedagógicas de salas de aula. El sistema castiga las resistencias, pero no alcanza a controlar las comprensiones y la conciencia afiatada de la identidad docente rural, que se desempeña en aulas multigrado. Por ello, mantiene la conciencia de que el sistema no representa al docente, por tener deficiencias en sus prácticas y concepciones acerca de su propio desempeño, que no está interesado en afrontar.

\section{Desafíos}

De nuestro estudio emanan algunos desafíos que, en nuestra opinión, deben asumir el Estado y Ministerio de Educación, así como los propios docentes rurales y el mundo académico, que compartimos a continuación, desde la voz del profesorado rural participante:

Visibilizar la realidad docente rural en la educación chilena, advirtiendo que los docentes son sometidos a un sistema de evaluación desajustado y descontextualizado a la realidad del trabajo que realizan.

Por ello, sensibilizar acerca de la falta de pertinencia de los instrumentos utilizados en la EDD en contextos de aula rural multigrado o en cursos combinados (confiabilidad y validez, desde el enfoque cualitativo).

Proponer mejoras para esta evaluación, escuchando e incorporando elementos que permitan visibilizar la práctica en aula multigrado.

Destacar la necesidad de implementar proyectos de desarrollo profesional docente, en el área 
específica de enseñanza rural multigrado: planificación, evaluación y desarrollo local.

\section{Proyecciones y nuevas preguntas para otras investigaciones}

El mundo académico en Chile escasamente contempla al profesorado rural en sus análisis sobre las diversas dimensiones y agentes del sistema escolar. Por ello interesa proponer algunas líneas de investigación posibles en esta temática con el fin de:

Reconocer y validar las buenas prácticas de aula rural, establecer cuáles arrojan los mejores desempeños y, respetando singularidades, proponer el estudio de estrategias para una evaluación docente que destaque las formas de preparar la enseñanza, la creación de un ambiente propicio para el aprendizaje, la enseñanza para el estudiantado en su totalidad y las responsabilidades profesionales que ese acto educativo requiere en la sala multigrado. Es decir, repensar una nueva forma de plasmar las exigencias de una evaluación que sea de verdad relevante, justa y útil.

Fortalecer la institución actual con la que cuenta el profesorado rural: los Microcentros. En nuestra opinión, estos deben ser potenciados como articuladores entre los resultados de las futuras eva- luaciones y las posibilidades de mejora y acompañamiento a los docentes de zonas rurales.

Constatar, en la voz del profesorado rural multigrado, las áreas que más se le dificultan y, a partir de esas declaraciones, proponer estrategias de perfeccionamiento a docentes en ejercicio y establecer recomendaciones a los programas universitarios de formación inicial docente. Todo ello tomando en cuenta la nueva propuesta de carrera docente que ha sido promulgada como ley. Este nuevo instrumento legal modificará las exigencias profesionales docentes en ejercicio, y es aquí donde nuevamente emerge la importancia del portafolio como instrumento que permite evaluar las prácticas docentes. El desafío es investigar acerca de las formas en que se plasmarán las nuevas exigencias y cuánto de ello reconoce la particularidad del trabajo docente en la escuela multigrado.

Por su enorme importancia, conocer el instrumento evaluativo de prácticas profesionales, ya que es a partir de los resultados que obtendrán en esta evaluación, sumados a sus especializaciones, como el profesorado puede avanzar en los diferentes tramos que propone la nueva carrera docente, recibir mejores sueldos y tener estabilidad como funcionario y en los cargos. Materias que en definitiva conllevan, por la sola condición de acceso, un grado extra de dificultad para los docentes de sectores rurales.

\section{Referencias}

Ávalos, B. (2004). Teacher regulatory forces and accountability policies in Chile: From public servants to accountable professionals. Research Papers in Education, 19(1), 67-85.

Álvarez, M. (2000). ¿Qué hay que evaluar de los docentes? CIDE. Recuperado de http://ww2.educarchile.cl/UserFiles/Pooo1\%5CFile\%5COu\%C3\%A9\%2ohay\%20que\%2oevaluar\%2oen\%2olos\%2odocentes.pdf

Bonifaz, R. (2011). Origen de la evaluación docente y su conexión con las políticas públicas en educación. En J. Manzi, R. González y Y. Sun (eds.), La evaluación docente en Chile (1 ed., pp. 13-34). Santiago: Pontificia Universidad Católica de Chile - Centro de Medición. 
Bonvecchio, M. (2006). Evaluación de los aprendizajes: manual para docentes. Buenos Aires: Novedades Educativas.

Campbell, R. J., Kyriakides, L., Muijs, R. D. y Robinson, W. (2003). Differential teacher effectiveness: towards a model for research and teacher appraisal. Oxford Review of Education, 29(3), 347-362.

Ctera-cnte, colegio de profesores, afutu-fenapes, lpp (2005) Las reformas educativas en los países del Cono Sur. Buenos Aires: CLAcso. Recuperado de http://biblioteca.clacso.edu.ar/ar/libros/edicion/reformas/reformas.html

Darling-Hammond, L. (2013). Getting teacher evaluation right. What really matters for effectiveness an improvement ( 1 ed.). Nueva York: Teacher College Press.

Eisenberg, N. V. (2008). The performance of teacher in Chilean public elementary schools: Exploring its relantionship with teacher backgrounds and student achievement, and its distributions across schools and municipalities. Disertación Doctoral, University of California Los Angeles.

Elola, N. y Toranzos, L. (2000). Evaluación educativa: una aproximación conceptual. Recuperado de http://uiap. dgenp.unam.mx/apoyo_pedagogico/proforni/antologias/EVALUACION\%2OEDUCATIVA.pdf

Fardella, C. (2012). Verdades sobre la docencia, efectos y consecuencias subjetivas de la evaluación docente en Chile. Revista de Psicología, 21(1).

García, E.y Cabero, J. (2011). Diseño y validación de un cuestionario dirigido a describir la evaluación en procesos de educación a distancia. Edutec-e, Revista Electrónica de Tecnología Educativa, 35. Recuperado de http:// www.edutec.es/revista/index.php/edutec-e/article/view/412

Gobierno de Chile (2011). Ley Núm. 20.501. Calidad y equidad de la educación. Recuperado de http://www.ley chile.cl/Navegar?idNorma=1022346

Gobierno de Chile (s.f.). Marco para la Buena Enseñanza. Ministerio de Educación. Recuperado de http://www. cpeip.cl/usuarios/cpeip/File/Documentos\%202011/MBE2008.pdf

Goe, L. (2007). The link between Teacher quality and student outcomes: A research synthesis. Washington: ERIC Institute of Education Sciences.

Goe, L., Bell, C.y Little, O. (2008). Approaches to evaluating teacher effectiveness: A research synthesis. Washington: National Comprehensive Center for Teacher Quality.

Goldhaber, D. (2008). Teachers matter, but effective teacher quality policies are elusive. En H. F. Ladd y E. B. Fiske (eds.), Handbook of Research in Education Finance and Policy (1 ed., pp. 146-165). Nueva York: Routledge.

Coggshall, J., Max, J. y Bassett, K. (2008, June). Key issue: Using Performance-based assesment to identify and support high- quality teachers. Washington, DC: National Comprehensive Center for Teacher Quality.

Howard, B. B.y Gullickson, A. R. (2010). Setting standards for teacher evaluation. En M. M. Kennedy (ed.), Teacher Assessment and the Quest for Teacher Quality (1 ed., pp. 337-354). San Francisco: Jossey-Bass. 
ISSN 0123-1294 | e-ISSN 2027-5358 | Educ.Educ. Vol. 20. No. 3 | Septiembre-diciembre de 2017 | pp. 364-381.

Universidad de La Sabana | Facultad de Educación

Lewis, W. D. y Young, T. V. (2013). The politics of accountability: Teacher education policy. Educational Policy, $27(2), 190-216$.

Manzi, J., González, R. y Sun, Y. (2011). La evaluación docente en Chile. Santiago: Pontificia Universidad Católica de Chile - Centro de Medición. Recuperado de http://www.mideuc.cl/libroed/pdf/La_Evaluacion_Docente_en_Chile.pdf

Milicic, N., Rosas, R., Scharager, J., García, M., Godoy, C. (2008). Diseño, construcción y evaluación de una pauta de observación de videos para evaluar calidad del desempeño docente. Revista Psykhe, 17(2), 79-90. Pontificia Universidad Católica de Chile.

Murillo Torrecilla, F. J., González de Alba, V. y Rizo Moreno, H. (2006). Evaluación del desempeño y carrera profesional docente. Un estudio comparado entre 50 paises de América y Europa (1 ed.). Santiago: orealc/unesco.

OECD (2013a). Synergies for better learning. An international perspective on evaluation and assessment. París: OECD.

OECD (2013b). Teachers for the $21^{\text {st }}$ Century. Using evaluation to improve teaching. París: OECD.

OREALC/UNesco (2007). Evaluación del desempeño y carrera profesional docente. Una panorámica de América y Europa. Chile: OREALC/UNESCO.

Peterson, K. (2000). Teacher evaluation. A comprehensive guide to new directions and practices (2 ed.). California: Corwin Press.

Porlán, R.y Rivero, A. (2000). El conocimiento de los profesores. Una propuesta formativa en el área de las ciencias. Sevilla: Diada.

Rodríguez, M., Hoffmann, C., Mackedanz, P. y Hoffmann, V. (2011). Como investigar cualitativamente. Entrevista y cuestionario. En Contribuciones a las Ciencias Sociales. eumed.ned. Recuperado de http://www.eumed. net/rev/cccss/11/

Sanders, W. L.y Horn, S.P. (1998). Research findings from the Tennessee Value-Added Assessment System (TVAAS ) database: Implications for educational evaluation and research. Journal of Personnel Evaluation in Education, 12(3), 247-256.

Sanders, W. L. y Rivers, J. C. (1996). Cumulative and residual effects on future academic achievment. Knoxville: University of Tennessee.

Santiago, P. (2013). Evaluación docente en Chile: Fortalezas y desafíos. Revisión de la OCDE de los marcos de evaluación para mejorar los resultados escolares. Seminario Organizado por Ministerio de Educación de Chile. Recuperado de http://www.docentemas.cl/docs/Presentacion_Revision_Ev_Docente_Chile_Paulo_Santiago_Recomendaciones2_o8112013.pdf 
Stronge, J. H. y Tucker, P. D. (2003). Teacher evaluation. Assessing and improving performance (1 ed.). Larchmont, NY: Eye on Education.

Taut, S., Santelices, V., Araya, C. y Manzi, J. (2010). Theory underlying a national teacher evaluation program. Evaluation and Program Planning, 33, 477-486.

Williamson, G. (2010). Todos en el mismo curso. Escuelas multigrado en la educación rural: reflexiones desde algunas experiencias internacionales. Revista Digital eRural, Educación, Cultura y Desarrollo Rural, 7(1314) (enero).

Williamson, G. (1999). Profesores rurales. Organización y reflexión pedagógica. Revista Docencia, 3(7), 50-55. Colegio de Profesores de Chile. 